\title{
Feasibility and acceptability of a technology-based, rural weight management intervention in older adults with obesity
}

John A. Batsis ${ }^{1,2^{*}}$, Curtis L. Petersen ${ }^{3}$, Matthew M. Clark ${ }^{4}$, Summer B. Cook ${ }^{5}$, David Kotz ${ }^{3}$, Tyler L. Gooding ${ }^{2}$, Meredith N. Roderka ${ }^{2}$, Rima I. Al-Nimr ${ }^{2}$, Dawna Pidgeon ${ }^{2}$, Ann Haedrich² ${ }^{2}$ K. C. Wright ${ }^{2}$, Christina Aquila ${ }^{2}$ and Todd A. Mackenzie ${ }^{3}$

\begin{abstract}
Background: Older adults with obesity residing in rural areas have reduced access to weight management programs. We determined the feasibility, acceptability and preliminary outcomes of an integrated technology-based health promotion intervention in rural-living, older adults using remote monitoring and synchronous video-based technology.

Methods: A 6-month, non-randomized, non-blinded, single-arm study was conducted from October 2018 to May 2020 at a community-based aging center of adults aged $\geq 65$ years with a body mass index (BMI) $\geq 30 \mathrm{~kg} / \mathrm{m}^{2}$. Weekly dietitian visits focusing on behavior therapy and caloric restriction and twice-weekly physical therapist-led group strength, flexibility and balance training classes were delivered using video-conferencing to participants in their homes. Participants used a Fitbit Alta HR for remote monitoring with data feedback provided by the interventionists. An aerobic activity prescription was provided and monitored.

Results: Mean age was $72.9 \pm 3.9$ years ( $82 \%$ female). Baseline anthropometric measures of weight, BMI, and waist circumference were $97.8 \pm 16.3 \mathrm{~kg}, 36.5 \pm 5.2 \mathrm{~kg} / \mathrm{m}^{2}$, and $115.5 \pm 13.0 \mathrm{~cm}$, respectively. A total of 142 participants were screened ( $n=27$ ineligible), and 53 consented. There were nine dropouts (17\%). Overall satisfaction with the trial $(4.7+0.6$, scale: 1 (low) to 5 (high)) and with Fitbit $(4.2+0.9)$ were high. Fitbit was worn an average of $81.7 \pm 19.3 \%$ of intervention days. In completers, mean weight loss was $4.6 \pm 3.5 \mathrm{~kg}$ or $4.7 \pm 3.5 \%(p<0.001)$. Physical function measures of 30-s sit-to-stand repetitions increased from $13.5 \pm 5.7$ to $16.7 \pm 5.9(p<0.001), 6$-min walk improved by $42.0 \pm 77.3 \mathrm{~m}$ ( $p=0.005)$ but no differences were observed in gait speed or grip strength. Subjective measures of latelife function improved (3.4 \pm 4.7 points, $p<0.001)$.

(Continued on next page)
\end{abstract}

\footnotetext{
* Correspondence: john.batsis@gmail.com

${ }^{1}$ Division of Geriatric Medicine, School of Medicine, and Department of

Nutrition, Gillings School of Global Public Health, University of North Carolina

at Chapel Hill, 5017 Old Clinic Building, Chapel Hill, NC 27599, USA

${ }^{2}$ Dartmouth-Hitchcock, Geisel School of Medicine, and The Dartmouth

Institute for Health Policy, Hanover, NH, USA

Full list of author information is available at the end of the article
}

(c) The Author(s). 2021 Open Access This article is licensed under a Creative Commons Attribution 4.0 International License, which permits use, sharing, adaptation, distribution and reproduction in any medium or format, as long as you give appropriate credit to the original author(s) and the source, provide a link to the Creative Commons licence, and indicate if changes were made. The images or other third party material in this article are included in the article's Creative Commons licence, unless indicated otherwise in a credit line to the material. If material is not included in the article's Creative Commons licence and your intended use is not permitted by statutory regulation or exceeds the permitted use, you will need to obtain permission directly from the copyright holder. To view a copy of this licence, visit http://creativecommons.org/licenses/by/4.0/ The Creative Commons Public Domain Dedication waiver (http://creativecommons.org/publicdomain/zero/1.0/) applies to the data made available in this article, unless otherwise stated in a credit line to the data. 
(Continued from previous page)

Conclusions: A technology-based obesity intervention is feasible and acceptable to older adults with obesity and may lead to weight loss and improved physical function.

Clinical trial registration: Registered on Clinicaltrials.gov \#NCT03104205. Registered on April 7, 2017. First participant enrolled on October 1st, 2018.

Keywords: Weight, Telehealth, Disparities

\section{Background}

Obesity rates in older adults have surpassed 35\% [1] of the population and have been associated with a two- to threefold higher risk of functional decline, [2] a 30\% higher risk of institutionalization [3] and mortality, [4] and \$1496 annualized higher health costs compared to healthy weight older adults [5]. Caloric restriction with aerobic and resistance training is central to weight loss and improving physical function, and leads to better quality of life [6]. Efficacy trials in frail, older adults have found that diet-exercise interventions were safe, improved function, reversed frailty, [7] improved gait speed, and delayed disability [8]. Yet, weight loss in older adults is not highly endorsed by clinicians, in part due to the conflicting literature of the safety of weight loss in older adults due to the obesity paradox [9]. Busy clinicians also do not have the time nor expertise to focus on weight loss efforts $[10,11]$. In fact, in Medicare beneficiaries, data showed low uptake despite coverage for obesity therapy, showing a need for new delivery systems [12].

Engaging in health promotion efforts requires proximity and frequent visits to medical facilities. This is often impossible for the 10 million older rural Americans whose obesity rates are 3.6-7\% higher, physical activity levels are $7 \%$ lower, and diet quality is lower, compared to urban residents [13]. Weight and nutrition are key priorities in Rural Healthy People 2020 [14] as rural care is affected by lower healthcare access, [15] a complex natural and built environment, [14] healthcare provider workforce shortages, and a lack of rural-specific programs [16]. Barriers also include a need for social connectedness, access, and program availability [17]. Previous weight loss efforts in rural areas generally have been achieved through in-person interventions, phone calls, community-health workers, or peer coaching [1821]. However, such health promotion efforts have rarely targeted this at-risk population which has significant comorbidity and where obesity can markedly impair physical function $[22,23]$.

Self-monitoring strategies, such as completing food records or exercise logs, predict initial and sustained weight loss, [24] but may be difficult to achieve in this population. Telehealth-including telemedicine (live, two-way, videoconferencing) or remote monitoring via wearable devices (providing bi-directional, synchronous, or asynchronous data feedback) - is a delivery strategy that addresses rural health barriers by overcoming the sparsity of resources, lack of available initiatives, and workforce shortages [25]. While evidence exists in pediatric and young adult populations, [26] it is unclear whether weight-management interventions for older rural-living adults using behavioral and engagement strategies are feasible to achieve the desired outcomes. As older adults' use of technology grows [27], using technology-based strategies can potentially be be used for clinical care. For instance, recent reviews have demonstrated that video-conferencing is feasible, acceptable and can effectively be used in older adults [28], as can fitness devices such as Fitbit for remote monitoring [29]. We designed a technology-based weight management intervention for rural older adults with obesity and evaluated its feasibility, acceptability and preliminary outcomes.

\section{Methods}

\section{Study design and setting}

This technology-based weight management intervention was a six-month, single-armed, weight-management intervention for older adults with obesity residing in rural New Hampshire and Vermont. Primary and secondary outcomes were evaluated at baseline $0,2,4$, and 6 months. The study was conducted from October 2018 to May 2020 with participants continually recruited. All testing activities were conducted on-site at the local Center for Health and Aging, a community-based resource center affiliated with Dartmouth-Hitchcock. The study was approved by the Dartmouth-Hitchcock Institutional Review Board. The trial was also registered on clinicaltrials.gov under NCT\#03104205.

\section{Study participants}

Participants were recruited from physician referral. Posters and tear-off cards were delivered to offices for distribution, and the senior author (JAB) presented the study to the local clinicians. There was a maximum of 10 participants per intervention group (led by one registered dietitian nutrition (RDN) and one licensed physical therapist $[\mathrm{PT}]$ at any one time). The electronic medical record (EMR) was used to assess selection criteria. Participants consisted of English-speaking communitydwelling older adults aged $65+$ with a BMI $>30 \mathrm{~kg} / \mathrm{m}^{2}$ that had access to high-speed Internet at home. 
Participants were excluded if they fulfilled any of the following EMR-listed diagnoses: end-stage congestive heart failure, renal insufficiency, dementia, or hepatic failure; a terminal/life-threatening illness; severe, uncontrolled psychiatric diagnosis; nursing home or hospital admission within the past 6 month; weight loss surgery; a lifeexpectancy < 6 months; on obesogenic medications; or > $5 \%$ weight loss in the past 6 months. A validated Callahan screen [30] for cognitive impairment, and the validated Older Americans Resources and Services questionnaire [31] for activities of daily living were administered by phone. Scores of $>3$ and $>6$, respectively, fulfilled eligibility criteria. Written permission from a primary care physician was required for participation. All participants came to the community-based Center for Health and Aging for consent and study assessments. Adverse events were monitored and documented on safety sheets.

\section{Study intervention}

Participants enrolled in a 26-week weight management program consisting of nutrition and exercise sessions delivered using a blend of synchronous, video-conferencing sessions with real-time communications and the use of remote monitoring using Fitbit and enhanced by periodic face-to-face interactions (Fig. 1). The nutrition sessions occurred either before or after the PT sessions. The intervention itself was based on structural elements of the social cognitive theory [32] and the technology acceptance model [33].

A RDN delivered 18 individual, 1:1 live videoconferencing nutrition sessions lasting $30 \mathrm{~min}$, and 7 onsite hourly group sessions (remotely if necessary) focusing on caloric restriction $(500-750 \mathrm{kCal} /$ day deficit, minimum $1200 \mathrm{kCal} /$ day), vitamin D (1,000 IU/day), protein intake $(1-1.2 \mathrm{~g} / \mathrm{kg} /$ day or $20 \%$ intake). Balanced, evidence-based individualized meal plans were guided by the HarrisBenedict equation [34] and indirect calorimetry (REEVue, Korr Medical). Motivational interviewing, goal-setting and behavioral strategies were used with written patient education materials throughout the intervention. Group onsite sessions summarized content and provided an opportunity for social connectedness. Weekly food records were reviewed, and attendance was monitored.
All study participants engaged in 75-min, twiceweekly, synchronous video-conferencing, group exercise sessions were led by a trained physical-therapist (PT) amounting to a total of 40 sessions. Over the course of the study, every 3-4 weeks, there was an on-site group session to promote social engagement amongst participants and interventionists, for a total of 7 additional sessions. The intensity of this program paralleled that of the American College of Sports Medicine recommendations for exercise in older adults [35]. Personalized resistance, flexibility and balance exercise plans were developed for all participants and conducted during each of these on-site and video-conferencing sessions. Resistance training [36] used adjustable weights and bands targeting major muscle groups $(30-45 \mathrm{~min} ; 8-12$ reps; 2 sets), increasing loads after 15 reps of full range of motion. Flexibility exercises included static stretches (1530 min; 30-60s each). Balance training focused on agility and coordination, and included static, dynamic, and vestibular exercises (15-30 min) [37]. Participants were also trained and guided to perform resistance, flexibility and balance exercise once weekly outside the study sessions (75 min). Progress was assessed and recorded by the physical therapist, monitored remotely, aimed at gradual workload increases for resistance training (Borg perceived exertion rate of 12 [somewhat hard]) [38]. Participants were advised to conduct the type of exercise, repetitions and sets during this specific time period. Each had a one-on-one 'video check-in' session weekly with the PT lasting 5 min during the intervention to adjust the proposed exercise plan and to assess progress in improving physical activity and function. Outside the structured exercise sessions, participants were guided to adopt a program of $150 \mathrm{~min} /$ week of moderate-intensity aerobic walking, in a minimum of 10-min bouts again, guided by the PT. [36] Hence, the total duration of study-related activities - aerobic, resistance, flexibility, balance - amounted to 375 min per week [36, 37, 39].

\section{Video Conferencing \& Remote Monitoring}

Synchronous, real-time communication with audio/ video-conferencing was delivered used a HIPAA compliant version of Zoom. The RDN used a webcam-enabled

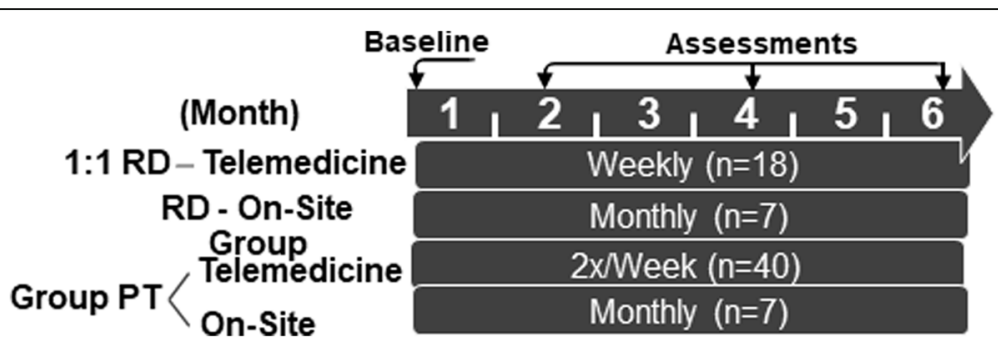

Fig. 1 Schematic of the Components of the Technology-Based Intervention 
laptop to conduct the 1:1 participant sessions. The PT delivered the exercise sessions in an office space, with their laptop connected to a 50 " television that permitted interaction and exercise delivery on a larger screen. A Logitech webcam and a wireless USB-microphone were used for video and audio. A Samsung Galaxy A Tab. 10.1 " tablet was given to participants. Written, step-bystep, picture-based instructions permitting them to connect the tablet to their home Wi-Fi and was then 'mirrored' to their personal television at home using an Amazon Firestick. At an orientation session, the research assistant demonstrated the setup; if connection issues persisted at home, they guided participants by phone. Tablet security was guided by institutional practices. Each participant was provided a Fitbit ALTA HR (Fitbit Inc., San Francisco, CA) to physical activity engagement throughout the six-month study. The research assistant demonstrated how to use and charge the device, and provided instructions on how to visualize data through the tablet-installed Fitbit-based app. Data was synchronized to a third-party software, Fitabase, which permitted data aggregation to the minute-level. Information was coded using a unique study identifier.

\section{Outcome measurements}

Our primary outcome measures were feasibility and acceptability of the intervention. Feasibility of our recruitment criteria (screening and enrollment), intervention completion rates, and attendance rates were assessed. Our target enrollment was 48 participants which assumed an estimate of a $20 \%$ dropout rate at 6-months and an attendance rate of $>75 \%$. We based these values on slightly more conservative estimates than our previous pilot study and other efficacy-based trials or reviews [7, 19, 40-43]. Adherence of Fitbit consisted of obtaining $>75 \%$ of available data [44] with at least $8 \mathrm{~h}$ of use [45]. Participant satisfaction surveys were conducted at home using RedCAP, a secure, web-based application that supports data capture for research (Additional file 1: Appendix 1). Acceptability was assessed using selfreport surveys, measured at study conclusion (range 15 , low to high). Acceptability of each measure was considered successful if the measure exceeded 4 of 5 points (> 80\%).

Baseline sessions were conducted by the research assistant and consisted of two technology training components (30 min each), two National Cancer Institute Automated Self-Administered-24 dietary assessment tool evaluations (30 min each) [46] and objective physical function assessments (45 min each - see below). An initial PT assessment permitted individualization of the exercise plan by gaining insights into their baseline performance status (45 $\mathrm{min}$ ), The co-primary effectiveness outcomes included changes in weight and in the 30-s sit-to-stand test (30STS). Weight was assessed using a standardized $\mathrm{A}+\mathrm{D}$ digital scale without shoes, jackets or heavy clothing and height was measured using a stadiometer. A 5\% change in weight is considered clinically significant [47]. The 30STS is a clinical construct of physical function that predicts falls and disability, is sensitive to change, and is highly correlated with quadriceps and leg press strength (test-retest reliability, $r=0.89$ in community dwelling older adults), leg performance $(r=$ 0.78 ) and 6-min walk ( $\mathrm{r}=0.53$, in pulmonary patients) [48-51]. Participants sat in a chair with a back, arms folded, and stood up/sat down as many times as they could for 30-s. A two-repetition increase is considered clinically significant [52]. Grip strength was assessed using a JAMAR handheld dynamometer, measured in both hands three times, alternating every $30 \mathrm{~s}$; maximum values were used in the analysis. Grip strength relates to upper and lower extremity strength, and predicts mobility disability (test-retest reliability, $\mathrm{r}=0.954$ in healthy elders) [53]. A clinically significant change in grip strength is $5 \mathrm{~kg}$ [53]. As a surrogate for submaximal exercise capacity, a 6-min walk in a long, $70 \mathrm{~m}$ corridor was conducted (test-retest, $\mathrm{r}=0.95$, in older adults without significant disease) [54]. A change in $30 \mathrm{~m}$ is considered clinically significant in older adults with multimorbidity [55]. Subjective measures of physical function was assessed using the 32-item function component of the Late-Life Function and Disability Instrument (LLFDI) [56]. This measure correlates with gait speed and lowerlimb function. Neither participants nor research staff were blinded to the objective outcome assessments.

\section{Statistical analysis}

Descriptive statistics evaluated feasibility and acceptability measures. Continuous variables are represented as means \pm standard deviation, and categorical values as counts (percent). An unpaired t-test or chi-square testing assessed differences between completers vs. dropouts (participants that did not complete the intervention). Intragroup comparisons of baseline and week 26 values were assessed using a paired t-test (or its non-parametric equivalent). Mixed-effects models (with a fixed effect of participant) evaluated longitudinal changes in weight, 30STS, 6-min walk and LLFDI adjusting for age and sex. We also evaluated the differences in objective physical function measures in participants losing $\geq 5 \%$ weight loss over time. Wear time was calculated using methods previously described [57]. All analyses were conducted using STATA v.15 or R (www.r-project.org) v.3.6. A p-value < 0.05 was considered statistically significant.

\section{Results}

Participant flow is presented in Additional file 1: Appendix 1 . The eligibility rate was $81 \%$, of which $53.9 \%$ 
declined participation. Of the 53 participants that enrolled (46.1\%), $44(83.0 \%)$ completed the intervention. Attendance rates for both the video and on-site visits were 77 and $78.2 \%$ for the physical therapy exercise sessions respectively, and 84 and $90.0 \%$, for the RDN-based visits respectively. Participants wore the Fitbit for an average of $81.7 \%$ of the days of the intervention and obtained an average of $4078 \pm 3819$ steps per day. The mean wear time was $8.3 \pm 3.8 \mathrm{~h}$ per day. During the last 2 months of the intervention, there were 7 participants where we were unable to capture data due to synchronization issues. Three of these seven were also unable to synchronize their devices during months 3 or 4 as well. There were no differences in the number of steps over time ( $p=0.83)$.

Satisfaction rates (Table 1) were high for both the overall intervention and for specific item-based questions related to the video-conferencing components. Participants were supportive of both the virtual-based physical therapy and dietary components of the intervention. Satisfaction related to the Fitbit was slightly lower than that of the video-conferencing.

There were no significant differences in baseline demographic characteristics in dropouts as compared to completers. Characteristics are outlined in Table 2 . The mean distance and time for participants to the center was $22.7 \pm 19.3$ miles and $24.0 \pm 20.3 \mathrm{~min}$ in enrollees. Table 3 outlines the anthropometric and objective outcome measures. Mean weight loss was $4.6 \pm 3.5 \mathrm{~kg}(4.7 \%)$, and 30 STS improved from $13.5 \pm 5.7$ to $16.7 \pm 5.9$ repetitions $(p<0.001)$ over six-months. Fifty-percent of the cohort had clinically significant improvements in weight $(n=22), 73 \%$ had at least a 2 repetition improvement in 30STS, and $41 \%$ had at least a $30 \mathrm{~m}$ improvement in 6-min walk. Changes in 6 - min walk were clinically significant $42.0 \pm 77.3 \mathrm{~m}(P=$ $0.005)$. Gait speed and grip strength did not change. Subjective measures of LLFDI also noted improvements in total, upper, basic lower, and advanced lower extremity function $(\mathrm{p}<0.001)$. Graphical representation and mixed-effect models are presented in Fig. 2 and absolute values are presented in Additional file 3: Appendix \#3. Participants kept on losing weight, improved their 30STS times, and improved their LLFDI scores across the different time points, while waist circumference and other functional measures plateaued earlier. Individuals losing $\geq 5 \%$ of their weight (pre/post) had significantly improved measures of objective physical function as compared to those not losing weight (Fig. 3). Our sensitivity analysis comparing the 33 participants with full follow-up data as compared to those without full data demonstrating differences in hyperlipidemia as a baseline comorbidity (14 [42.4\%] vs. 1 [11\%], $p<0.001)$. There were no differences in primary outcomes of weight change or sit-to-stand repetitions (data not shown).

There was one serious adverse event consisting of an emergency room episode for newly diagnosed atrial fibrillation. Adverse events that were definitely related to the study predominantly consisted of musculoskeletal complaints $(n=6)$, skin rash due to Fitbit $(n=1)$, hypoglycemia $(n=1)$, and upset stomach $(n=1)$ (Additional file 4: Appendix \#4).

\section{Discussion}

The findings from this technology-based intervention are timely in that it provides preliminary feasibility, acceptability, and outcomes of technology-based strategies for older adults with obesity residing in rural areas. The intervention not only led to improvements in weight, but also demonstrated key improvements in physical function measures such as 30STS and 6-min walk, both key markers of disability and independence. Notably, this intervention was conducted prior to the pandemic of COVID-19; hence, it is plausible that this technologybased intervention would have even greater appeal to an at-risk older adult population.

Our approach provided informative feasibility data that could be helpful in structuring a large-scale intervention. First, we provide that older adults can engage in the use of remote monitoring and video-conferencing, dispelling major misconceptions that this group has difficulty in using technology [58]. In fact, acceptability was high and none of the nine participants that dropped out was because of the technology itself. Second, our recruitment strategies demonstrated that our inclusion/exclusion criteria were appropriate in that $<20 \%$ were ineligible. Evaluating the potential factors that precluded involvement showed that there continues to be a slight digital divide in internet accessibility; a future trial should provide internet access to prospective participants (e.g., through a Wi-Fi hotspot). All the other negative screening criteria were aligned with medical appropriateness for weight loss in a high-risk population with medical conditions. Third, those eligible who declined (e.g., family caregiving, worry about using technology, having other commitments, or had no interest) cited the common issues faced by recruiting older adults in clinical research [59]. Caregiving efforts often impair health promotion efforts [60], and our findings confirm such observations. The program's intensity and number of sessions may be a factor, suggesting that a future trial should reduce the number of sessions. Participants felt supported by the technical support, and that our procedures allowed engagement and monitoring throughout the study.

Recruitment of older adults with multiple chronic conditions is often challenging and hence they are under- 
Table 1 Baseline Characteristics of the Telemedicine Cohort

\begin{tabular}{|c|c|c|c|c|}
\hline & Overall & Completers & Dropouts & P-value \\
\hline & $N=53$ & $N=44$ & $N=9$ & \\
\hline Age, years & $72.9 \pm 3.9$ & $73.2 \pm 3.9$ & $71.4 \pm 3.8$ & 0.20 \\
\hline Female Sex & $37(69.8)$ & $32(72.7)$ & $5(55.6)$ & 0.30 \\
\hline Education & & & & 0.17 \\
\hline High school & $7(13.2)$ & $7(15.9)$ & 0 & \\
\hline Some College & $15(28.3)$ & $14(31.8)$ & $1(11.1)$ & \\
\hline College Degree & $15(28.3)$ & $12(27.3)$ & $3(33.3)$ & \\
\hline Post-College Degree & $16(30.2)$ & $11(25.0)$ & $5(55.6)$ & \\
\hline Income & & & & 0.45 \\
\hline Less than $\$ 25,000$ & $10(18.9)$ & $9(20.5)$ & $1(11.1)$ & \\
\hline$\$ 25,000$ to $\$ 49,999$ & $10(18.9)$ & $7(15.9)$ & $3(33.3)$ & \\
\hline$\$ 50,000$ to $\$ 74,999$ & $11(20.8)$ & $11(25.0)$ & 0 & \\
\hline$\$ 75,000$ to $\$ 99,999$ & $13(24.5)$ & $10(22.7)$ & $3(33.3)$ & \\
\hline$\$ 100,000$ to $\$ 199,999$ & $8(15.1)$ & $6(13.6)$ & $2(22.2)$ & \\
\hline$\$ 200,000$ or more & $1(1.9)$ & $1(2.3)$ & 0 & \\
\hline \multicolumn{5}{|l|}{ Insurance } \\
\hline Medicaid & $1(1.9)$ & 0 & $1(11.1)$ & 0.15 \\
\hline Medicare & $48(90.6)$ & $41(93.2)$ & $7(77.8)$ & 0.03 \\
\hline Private & $32(60.4)$ & $25(56.8)$ & $7(77.8)$ & 0.24 \\
\hline Smoking Status & & & & 0.78 \\
\hline Current & $1(1.92)$ & $1(2.3)$ & 0 & \\
\hline Former & $21(40.4)$ & 17 (38.6) & $4(50.0)$ & \\
\hline Never & $30(57.7)$ & $26(59.1)$ & $4(50.0)$ & \\
\hline Marital Status & & & & 0.53 \\
\hline Married & $35(66.0)$ & $28(63.6)$ & $7(77.8)$ & \\
\hline Widow & $5(9.4)$ & $5(11.4)$ & 0 & \\
\hline Single & $13(24.5)$ & $11(25.0)$ & $2(22.2)$ & \\
\hline Distance to Center, miles & $22.7 \pm 19.3$ & $24.0 \pm 20.3$ & $15.9 \pm 11.1$ & 0.25 \\
\hline Distance to Center, minutes & $29.6 \pm 20.6$ & $31.1 \pm 21.9$ & $22.3 \pm 10.4$ & 0.25 \\
\hline \multicolumn{5}{|l|}{ Co-Morbidities } \\
\hline Anxiety & $5(9.4)$ & $4(9.0)$ & $1(11.1)$ & 0.85 \\
\hline COPD & $4(7.5)$ & $3(6.8)$ & $1(11.1)$ & 0.66 \\
\hline Depression & $12(22.6)$ & $12(27.3)$ & 0 & 0.08 \\
\hline Diabetes & $14(26.4)$ & $14(31.8)$ & 0 & 0.05 \\
\hline Fibromyalgia & $2(3.8)$ & $2(4.6)$ & 0 & 0.51 \\
\hline High Cholesterol & 19 (39.9) & $15(34.1)$ & $4(44.4)$ & 0.56 \\
\hline Hypertension & $38(71.7)$ & $31(70.5)$ & $7(77.8)$ & 0.66 \\
\hline Osteoarthritis & $19(35.9)$ & $16(36.4)$ & $3(33.3)$ & 0.86 \\
\hline Sleep Apnea & 21 (39.6) & $18(40.9)$ & $3(33.3)$ & 0.67 \\
\hline Stroke & $2(3.8)$ & $1(2.3)$ & $1(11.1)$ & 0.21 \\
\hline
\end{tabular}

All values represented are means \pm standard deviation or counts (\%). P-value represents difference between completers and participants that dropped out from the 6-month intervention 
Table 2 Satisfaction Responses with the Technology-Based Intervention

\begin{tabular}{|c|c|c|}
\hline Overall Intervention & Mean & Range \\
\hline Overall Satisfaction & $4.7 \pm 0.6$ & $3-5$ \\
\hline Recommend the technology-based intervention to a family member & $5.0 \pm 0.2$ & $4-5$ \\
\hline Helpful for patients living in rural areas & $4.9 \pm 0.4$ & $3-5$ \\
\hline Helpful in assisting in achieving goals & $4.7 \pm 0.7$ & $3-5$ \\
\hline Beneficial and worth your time & $4.8 \pm 0.6$ & $3-5$ \\
\hline Video-Based Satisfaction Measures & Mean & Range \\
\hline Satisfaction with video-conferencing device & $4.4 \pm 1.0$ & $2-5$ \\
\hline Video-conferencing assist in achieving goals & $4.7 \pm 0.6$ & $3-5$ \\
\hline Video easy to use without much difficulty & $4.5 \pm 0.7$ & $3-5$ \\
\hline Physical Therapy & Mean & Range \\
\hline Program Delivery was useful & $4.7 \pm 0.6$ & $3-5$ \\
\hline Length of session & $4.8 \pm 0.5$ & $3-5$ \\
\hline Number of Sessions & $4.7 \pm 0.5$ & $3-5$ \\
\hline Nutrition & Mean & Range \\
\hline Program Delivery was useful & $4.9 \pm 0.3$ & $4-5$ \\
\hline Length of session & $4.9 \pm 0.3$ & $4-5$ \\
\hline Number of Session & $4.8 \pm 0.5$ & $3-5$ \\
\hline Willingness for Remote Intervention & N (\%) & N (\%) \\
\hline Physical therapy & $37(84.1)$ & - \\
\hline Dietitian sessions & $40(90.9)$ & - \\
\hline Location & Mean & Range \\
\hline Easier to perform activity & $4.1 \pm 0.9$ & $3-5$ \\
\hline Adequate support for Fitbit & $4.2 \pm 1.0$ & $1-5$ \\
\hline Satisfaction Questions on Fitbit & Mean & Range \\
\hline Overall satisfaction with Fitbit ${ }^{a}(n=2)$ & $4.2 \pm 0.9$ & $2-5$ \\
\hline Easy to use without much difficulty & $4.3 \pm 0.9$ & $2-5$ \\
\hline Real-time feedback helpful in promoting physical activity & $4.0 \pm 1.0$ & $2-5$ \\
\hline Helpful in achieving your goal & $3.8 \pm 1.0$ & $1-5$ \\
\hline
\end{tabular}

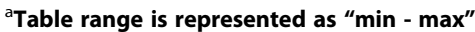

represented in clinical trials. In fact, the National Institute of Health's Inclusion of the Lifespan policy [61] promotes older adult's participation in research. While our study focused on older adults, our efforts were successful in a short period of time as recruitment occurred within a span of 1 year. Our retention rates were favorable and aligned with those of other obesity trials $[7,40]$. We recognize that participants may have been a motivated group willing to engage in health promotion efforts; those enrolled had goals of enhancing one's health. Yet, we do caution that sampling issues should be considered when interpreting our findings as this technology-based intervention may not be feasible in those not motivated or those with additional mobility impairments.

The adherence rates in this intervention were high, both using the remote monitoring device but also with our video-visits, suggesting that rural barriers to care delivery may potentially be overcome using our approach. A major shortcoming was our inability to synchronize Fitbit device data remotely; this could only occur during the on-site, in-person sessions. Participants had issues with the Fitbit application, which occasionally logged out and did not pair up with their Fitbit device; to our knowledge, this was not a Wi-Fi issue. This is encouraging as future devices have demonstrated better (and easier) connectivity to different platforms and a future study should address such connectivity issues. As such, this was a lost opportunity for capturing data and engaging participants. Our survey findings suggested a greater needed for continuous feedback to enhance engagement efforts [29]. Future studies should consider the ease of synchronization both for participants, but also for study personnel, remotely. 
Table 3 Preliminary Outcome Measures of Completers $(n=44)$

\begin{tabular}{|c|c|c|c|c|c|}
\hline & Baseline $(\mathrm{N}=44)$ & Week $26(\mathrm{~N}=44)$ & Difference $(\mathrm{N}=44)$ & Percent Change & $p$ value \\
\hline \multicolumn{6}{|l|}{ Anthropometric } \\
\hline Weight, kg & $97.8 \pm 16.3$ & $93.2 \pm 15.8$ & $-4.6 \pm 3.5$ & $-4.7 \pm 3.5$ & $<0.001$ \\
\hline $\mathrm{BMI}, \mathrm{kg} / \mathrm{m}^{2}$ & $36.5 \pm 5.2$ & $34.7 \pm 5.4$ & $-1.8 \pm 1.4$ & $-5.1 \pm 4.1$ & $<0.001$ \\
\hline Waist circumference, cm & $115.5 \pm 13.0$ & $112.8 \pm 11.9$ & $-2.6 \pm 5.4$ & $-2.1 \pm 4.7$ & 0.01 \\
\hline Waist to hip ratio & $0.926 \pm 0.081$ & $0.935 \pm 0.075$ & $0.009 \pm 0.0421$ & $1.1 \pm 4.7$ & 0.21 \\
\hline \multicolumn{6}{|l|}{ Objective Measures } \\
\hline 30-s Sit To Stand, repetitions & $13.5 \pm 5.7$ & $16.7 \pm 5.9$ & $3.1 \pm 4.2$ & $26.1 \pm 35.6$ & $<0.001$ \\
\hline${ }^{\mathrm{a}} 6$ min walk test, $\mathrm{m}$ & $387.0 \pm 94.9$ & $425.0 \pm 91.7$ & $42.0 \pm 77.3$ & $15.1 \pm 28.2$ & 0.005 \\
\hline${ }^{a}$ Gait Speed, sec & $1.05 \pm 0.23$ & $1.04 \pm 0.20$ & $-0.34 \pm 0.14$ & $-1.378 \pm 11.6$ & 0.16 \\
\hline aGrip Strength, kg & $24.8 \pm 9.9$ & $25.9 \pm 10.6$ & $1.2 \pm 7.0$ & $14.7 \pm 51.4$ & 0.33 \\
\hline \multicolumn{6}{|l|}{ Subjective Measures } \\
\hline \multicolumn{6}{|l|}{ Late-Life Functionality } \\
\hline Total & $59.8 \pm 8.2$ & $63.2 \pm 9.3$ & $3.4 \pm 4.7$ & - & $<0.001$ \\
\hline Upper extremity & $78.4 \pm 13.4$ & $81.0 \pm 12.7$ & $2.6 \pm 7.7$ & - & $<0.001$ \\
\hline Basic lower extremity & $72.9 \pm 12.5$ & $79.3 \pm 14.6$ & $6.4 \pm 11.1$ & - & $<0.001$ \\
\hline Advanced lower extremity & $48.7 \pm 12.3$ & $53.3 \pm 14.5$ & $4.6 \pm 8.2$ & - & $<0.001$ \\
\hline Fitbit Activity Measures & Mean & Range & Median & & \\
\hline$\%$ days worn ${ }^{*}$ & $81.7 \pm 19.3$ & $35.1-100$ & 89.1 & - & - \\
\hline Steps per day & $4078 \pm 3819$ & $0-29,884$ & 3443 & - & - \\
\hline
\end{tabular}

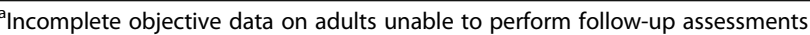

Worn is defined as recorded $\geq 100$ steps that day

Our staff was trained to assess adverse events using remote delivery, and repeatedly communicated with primary-care physicians. The number of musculoskeletalrelated events was high; none were classified as serious. Hypoglycemia occurred in one participant; none experienced undue coronary events nor hypotension, all potential consequences of weight loss. Future studies should continue to involve participant's primary-care providers, considering the high-risk population we are targeting, as they have knowledge of their medical history and can easily address medication changes as a result of weight loss.

Evidence-based strategies for video-conferencing and remote monitoring in a rural-living older populations are clearly lacking. A systematic review previously published by our group found a dearth of clinical trials; however, in those included, telemedicine could enhance older adults' outcomes despite a high degree of trial bias [28]. Telemedicine may lead to higher weight loss [62] and may be cost-effective [63]. Yet, a separate systematic review found that telemedicine-delivered interventions reduce BMI, only one focused on older adults (IDEATel) [64]. TeleMOVE (in Veterans) found higher weight loss (vs. controls or in-person), [65] but was not focused on older adults. A six-month telemedicine study in cardiac rehabilitation noted higher weight loss and patient activation [66]. Telemedicine can also be useful for weight maintenance, and is feasible in persons at risk for falls, sarcopenia, those planning bariatric surgery, and those with sensory impairments [67-70]. Usability issues may impact the use of telemedicine; hence the need for a platform that can be navigated easily with technical support [71]. In addition to the recent need to use alternative delivery systems due to the coronavirus pandemic, our findings provide timely data on the acceptability of an intervention that limits person-to-person contact. The implications of considering such an intervention on a larger scale in a time when social distancing and isolation are rampant amongst older adults [72] cannot be understated. In fact, this proposed technology-based intervention can be easily delivered in the midst of the crisis our society is facing to maintain and preserve function through health promotion efforts.

As older adults' use of technology grows (in 2019, Internet use was $>70 \%$, smartphone use $>53 \%$ ), using wearable technology such as Fitbit can feasibly and practically be used as clinical tools [27]. Such monitoring is promising and may elicit diet-exercise behavior change by improving self-management, tracking, social support, and goal setting; yet, results in younger populations are mixed and short-term [73, 74]. The IDEA trial found lower weight loss in the mHealth arm (vs. standard), in contrast to Spring's trial of health coaching and digital assistants [75, 76]. Using wearables with coaching in older adults with peripheral artery disease [77] did not 


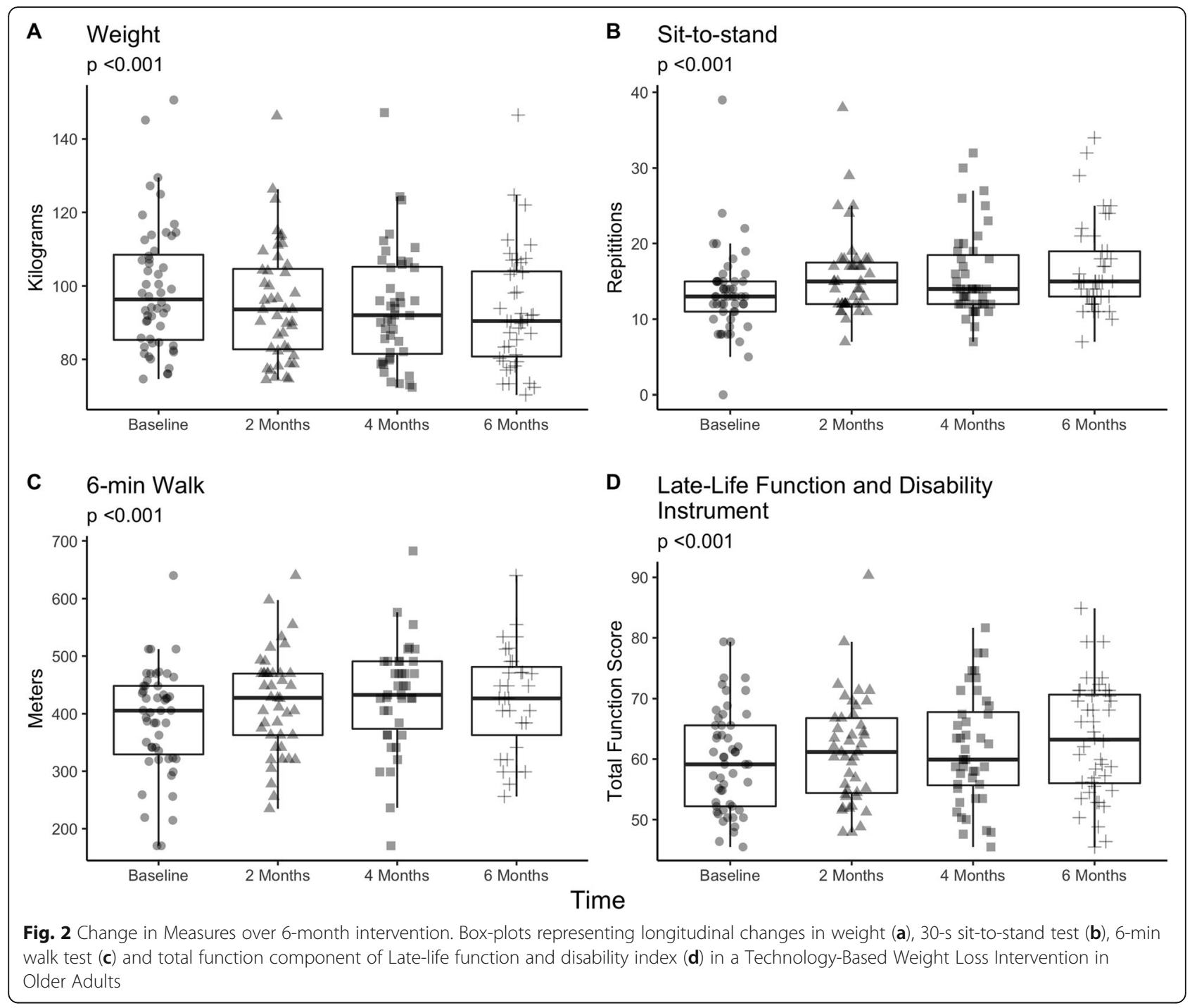

improve 6-min walk distance. While systematic reviews advise using mHealth in obesity trials, [78] it may be useful to couple mHealth with other modalities, including video-conferencing, to enhance care over in-person delivery [79].

The preliminary outcome findings near the threshold for clinically significant weight loss [47]. The statistically significant improvements in 30STS and 6-min walk test distances are clinically relevant. These improvements are known to be related to improved quality of life, physical function and are related to lower mortality. We recognize that this intervention may not necessarily be suitable for certain seniors who may be uncomfortable with technology or who do not have the necessary readiness to change to participate. There may be unknown biological factors that limit the intervention's effectiveness. Future studies need to better evaluate the characteristics of individuals enrolling in this intervention to provide a personalized approach to treatment.
This study is not without limitations. This was a nonrandomized, feasibility study without a control group with repeated measures at four time points strengthened our internal validity and enabling our ability to make an inference on change over time. Second, there was little ethnic diversity in a predominantly female cohort. Third, other rural areas may have different broadband capabilities or access to healthcare settings have different sociodemographic characteristics, thus may not be representative of rural dwellers. The counties served in rural New Hampshire had $>96 \%$ availability of internet connectivity [80]. Fourth, follow-up grip strength, gait speed, and 6-min walk were not obtained in 11 participants (25\% of completers) due to the COVID-19 pandemic. Our sensitivity analysis compared both baseline characteristics and outcomes of weight change and 30STS finding no differences suggesting we would have similar results. Fifth, the Fitbit used is now obsolete; emerging technologies have enhanced accuracy, precision 


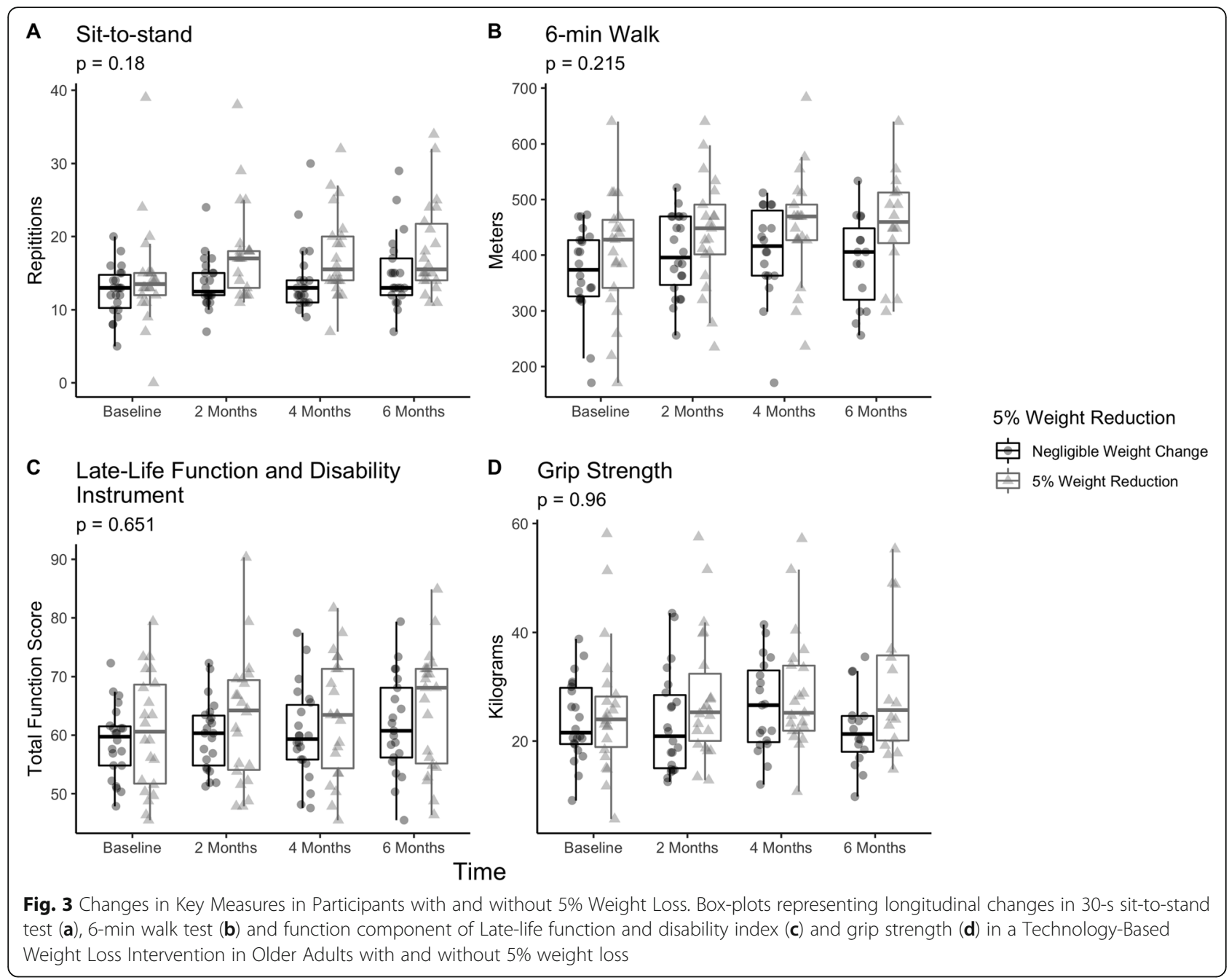

and usability. Sixth, the study's intensity was generally high but its individual components (nutrition and exercise), align with other community-based interventions. Future research should identify the necessary intensity to achieve weight loss and improved physical function, as limiting the number of sessions could enhance longer-term intervention compliance. Last, challenges in Fitbit synchronization observed in the last 2 months of our study reduced the reliability of our step counts.

Ongoing rural weight loss programs focus mainly on behavior or nutrition, rather than including exercise, and do not target seniors. MOVE-UP's use of community health workers delivering a diet and selfguided exercise intervention in older adults may provide insights into rural geriatric obesity care [20]. Issues related to geographic isolation among underserved, rural older adults using technology are not being addressed in these trials. Our proposed intervention potentially can contribute to rural health care-delivery science of innovative, effective, and pragmatic health-promoting activities that overcome barriers to rural healthcare in this population by improving physical function. Future trials are poised to address service gaps by using technology - and emerging telehealth regulations may permit billing, irrespective of site. Cost-effectiveness and economic analysis studies could be beneficial and helpful in the future. Telehealth is increasingly possible in older adults, with over $73-85 \%$ having access to broadband. The use of novel and practical technologies may permit integration of this technology-based intervention into health-care systems and clinical practice to ultimately improve quality and provide a scalable opportunity for widespread dissemination in rural America.

\section{Conclusions}

This technology-based, video-monitoring and remote monitoring intervention is feasible, acceptable, and demonstrates favorable outcomes by overcoming the limitations of existing geriatric weight-loss trials, overcoming a need for proximity to medical facilities. 


\section{Supplementary Information}

The online version contains supplementary material available at https://doi. org/10.1186/s12877-020-01978-x.

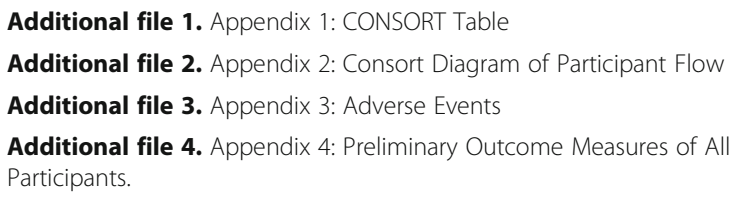

\section{Abbreviations}

BMI: Body mass index; 30STS: 30-s sit-to-stand; EMR: Electronic medical record; LLFDI: Late-life function and disability instrument; PT: Physical therapist; RDN: Registered dietitian nutritionist

\section{Acknowledgements}

We thank the following persons who assisted in the execution of study activities: Emma Brooks, Vanessa Brown, Molly Caisse, Peter DiMilia, Lori Fortini, Fredric Glazer, Aimee Burnett Greeley, Gregory Hagley, Chizuko Horiuchi, Shelley Hunevan, Diane Kelecy, Mary Lowry, Xingyi Li, Rebecca Masutani, Kristina Moses, Janice Montgomery, Meredith Roderka. A specia thanks to Dr. Ellen Flaherty for her support, Stephen Bartels, Martha Bruce, Francisco Lopez-Jimenez, Michael Jensen for their mentorship.

\section{Authors' contributions}

All authors have read and approved the manuscript. JAB: conceived, designed, analyzed, drafted and approved final version. CLP: analysis, critically reviewed and approval of final version. MMC: conceived, designed, critically reviewed and approved final version. SBC: conceived, designed, critically reviewed and approved final version. DK: conceived, designed, critically reviewed and approved final version. TLG: analysis, critically reviewed and approved final version. MNR: drafted, critically reviewed and approved final version. RIA: designed, critically reviewed and approved final version. DP: conceived, designed, and approved final version. AH: critically reviewed and approved final version. KCW: critically reviewed and approved final version. CA: critically reviewed and approved final version. TAM: conceived, designed, analyzed, and approved final version

\section{Funding}

Dr. Batsis' research reported in this publication was supported in part by the National Institute on Aging and Office of Dietary Supplements of the National Institutes of Health under Award Number K23AG051681 and R01AG067416. Support was also provided by the Dartmouth Health Promotion and Disease Prevention Research Center supported by Cooperative Agreement Number U48DP005018 from the Centers for Disease Control and Prevention, and the The Dartmouth Clinical and Translational Science Institute, under award number UL1TR001086 from the National Center for Advancing Translational Sciences (NCATS) of the National Institutes of Health (NIH). Dr. Batsis has been a consultant for Dinse, Knapp, MCAndrew, LLC, Legal Firm and for Turner Farms, LLC. He also has received funding from the Patient Centered Oriented Research Institute. Mr. Petersen is supported by the Burroughs-Wellcome Fund: Big Data in the Life Sciences at Dartmouth. Dr. Kotz is supported by the National Science Foundation awards CNS-1314281 and CNS-1619970 and by NIH/NIDA P30DA029926. Dr. Clark is a consultant for Roche Diabetes Care GmbH. The content is solely the responsibility of the authors and does not necessarily represent the official views of the National Institutes of Health or other sponsors, or represent the official position of the Centers for Disease Control and Prevention. The funders had no role in the design, conduct or analysis of the study.

\section{Availability of data and materials}

The datasets generated and/or analyzed during the current study are not publicly available due to data use/privacy issues but are available from the corresponding author on reasonable request.

\section{Ethics approval and consent to participate}

This study was approved by the Dartmouth-Hitchcock Institutional Review Board (\#28905). Informed consent was obtained by all participants in this study in a written manner, per institutional regulations.
Consent for publication

Not applicable.

\section{Competing interests}

None.

\section{Author details}

${ }^{1}$ Division of Geriatric Medicine, School of Medicine, and Department of Nutrition, Gillings School of Global Public Health, University of North Carolina at Chapel Hill, 5017 Old Clinic Building, Chapel Hill, NC 27599, USA. ${ }^{2}$ Dartmouth-Hitchcock, Geisel School of Medicine, and The Dartmouth Institute for Health Policy, Hanover, NH, USA. ${ }^{3}$ Dartmouth College, Hanover, $\mathrm{NH}$, USA. ${ }^{4}$ Mayo Clinic Rochester, Department of Psychiatry and Psychology, and Division of Endocrinology, Rochester, MN, USA. ${ }^{5}$ University of New Hampshire, Durham, NH, USA.

Received: 7 August 2020 Accepted: 21 December 2020

Published online: 12 January 2021

\section{References}

1. Hales CM, Fryar CD, Carroll MD, Freedman DS, Ogden CL. Trends in obesity and severe obesity prevalence in US youth and adults by sex and age, 2007-2008 to 2015-2016. JAMA. 2018;319(16):1723-5.

2. Jensen GL, Friedmann JM. Obesity is associated with functional decline in community-dwelling rural older persons. J Am Geriatr Soc. 2002;50(5):91823.

3. Zizza CA, Herring A, Stevens J, Popkin BM. Obesity affects nursing-care facility admission among whites but not blacks. Obes Res. 2002;10(8):81623.

4. Flegal KM, Kit BK, Orpana H, Graubard BI. Association of all-cause mortality with overweight and obesity using standard body mass index categories: a systematic review and meta-analysis. JAMA. 2013;309(1):71-82.

5. Musich S, MacLeod S, Bhattarai GR, Wang SS, Hawkins K, Bottone FG Jr, Yeh CS. The impact of obesity on health care utilization and expenditures in a Medicare supplement population. Gerontol Geriatr Med. 2016;2: 2333721415622004

6. Batsis JA, Gill LE, Masutani RK, Adachi-Mejia AM, Blunt HB, Bagley PJ, LopezJimenez F, Bartels SJ. Weight loss interventions in older adults with obesity: a systematic review of randomized controlled trials since 2005. J Am Geriatr Soc. 2017:65(2):257-68

7. Villareal DT, Aguirre L, Gurney AB, Waters DL, Sinacore DR, Colombo E, Armamento-Villareal R, Qualls C. Aerobic or resistance exercise, or both in dieting obese older adults. N Engl J Med. 2017;376:1943-55.

8. Nicklas BJ, Brinkley TE, Houston DK, Lyles MF, Hugenschmidt CE, Beavers KM, Leng $X$. Effects of caloric restriction on cardiorespiratory fitness, fatigue, and disability responses to aerobic exercise in older adults with obesity: a randomized controlled trial. J Gerontol A Biol Sci Med Sci. 2019;74(7):108490.

9. Strandberg TE, Strandberg AY, Salomaa W, Pitkala KH, Tilvis RS, Sirola J, Miettinen TA. Explaining the obesity paradox: cardiovascular risk, weight change, and mortality during long-term follow-up in men. Eur Heart J. 2009; 30(14):1720-7

10. Aleem S, Lasky R, Brooks WB, Batsis JA. Obesity perceptions and documentation among primary care clinicians at a rural academic health center. Obes Res Clin Pract. 2015;9(4):408-15.

11. Kahan S, Kushner RF. Obesity Medicine: A Core Competency for Primary Care Providers. Med Clin North Am. 2018;102(1):xvii-xix.

12. Dewar S, Bynum J, Batsis JA. Uptake of Obesity Intensive Behavioral Treatment Codes in Medicare Beneficiaries, 2012-2015. J Gen Intern Med. 2020;35(1):368-70. https://doi.org/10.1007/s11606-019-05437-1.

13. Trivedi T, Liu J, Probst J, Merchant A, Jhones S, Martin AB. Obesity and obesity-related behaviors among rural and urban adults in the USA. Rural Remote Health. 2015;15(4):3267.

14. Bolin JN, Bellamy GR, Ferdinand AO, Vuong AM, Kash BA, Schulze A Helduser JW. Rural healthy people 2020: new decade, Same Challenges. J Rural Health. 2015;31(3):326-33.

15. Foley DJ, Heimovitz HK, Guralnik JM, Brock DB. Driving life expectancy of persons aged 70 years and older in the United States. Am J Public Health 2002;92(8):1284-9. 
16. National Academies of Sciences E, Medicine. Achieving Rural Health Equity and Well-Being: Proceedings of a Workshop. Washington, DC: The National Academies Press; 2018.

17. Batsis JA, Naslund JA, Zagaria AB, Kotz D, Dokko R, Bartels SJ, CarpenterSong E. Technology for Behavioral Change in rural older adults with obesity. J Nutr Gerontol Geriatr. 2019;38(2):130-48.

18. Befort CA, Klemp JR, Austin HL, Perri MG, Schmitz KH, Sullivan DK, Fabian CJ. Outcomes of a weight loss intervention among rural breast cancer survivors. Breast Cancer Res Treat. 2012;132(2):631-9.

19. Batsis JA, Petersen CL, Clark MM, Cook SB, Lopez-Jimenez F, Al-Nimr RI, Pidgeon D, Kotz D, Mackenzie TA, Bartels SJ. A Weight Loss Intervention Augmented by a Wearable Device in Rural Older Adults With Obesity: A Feasibility Study. J Gerontol A Biol Sci Med Sci. 2021;76(1):95-100. https:// doi.org/10.1093/gerona/glaa115.

20. Venditti EM, Zgibor JC, Vander Bilt J, Kieffer LA, Boudreau RM, Burke LE, Glynn NW, Jakicic JM, Smith KJ, Semler LN, et al. Mobility and Vitality Lifestyle Program (MOVE UP): A Community Health Worker Intervention for Older Adults With Obesity to Improve Weight, Health, and Physical Function. Innov Aging. 2018;2(2):igy012.

21. Leahey TM, Wing RR. A randomized controlled pilot study testing three types of health coaches for obesity treatment: professional, peer, and mentor. Obesity (Silver Spring). 2013;21(5):928-34.

22. Batsis JA, Zbehlik AJ, Barre LK, Bynum JP, Pidgeon D, Bartels SJ. Impact of obesity on disability, function, and physical activity: data from the osteoarthritis initiative. Scand J Rheumatol. 2015;44(6):495-502.

23. Visser M. Obesity, sarcopenia and their functional consequences in old age Proc Nutr Soc. 2011;70(1):114-8.

24. Wing RR, Crane MM, Thomas JG, Kumar R, Weinberg B. Improving weight loss outcomes of community interventions by incorporating behavioral strategies. Am J Public Health. 2010;100(12):2513-9.

25. Gamm LD, Hutchison LL, Dabney BJ, Dorsey AM. Rural health people 2010 a companion document to health people 2010, vol. 1. College Station: The Texas A\&M University System Health Science Center, School of Rural Public Health, Southwest Rural Health Research Center; 2003.

26. Davis AM, Sampilo M, Gallagher KS, Dean K, Saroja MB, Yu Q, He J, Sporn N. Treating rural paediatric obesity through telemedicine vs. telephone: outcomes from a cluster randomized controlled trial. J Telemed Telecare. 2016;22(2):86-95.

27. Pew Internet Survey. 2019. https://www.pewresearch.org/internet/chart/ internet-use-by-age/. Accessed 9 Jan 2020.

28. Batsis JA, DiMilia PR, Seo LM, Fortuna KL, Kennedy MA, Blunt HB, Bagley PJ Brooks J, Brooks E, Kim SY, Masutani RK, Bruce ML, Bartels SJ. Effectiveness of Ambulatory Telemedicine Care in Older Adults: A Systematic Review. J Am Geriatr Soc. 2019 Aug;67(8):1737-49. https://doi.org/10.1111/jgs.15959. Epub 2019.

29. Batsis JA, Naslund JA, Gill LE, Masutani RK, Agarwal N, Bartels SJ. Use of a wearable activity device in rural older obese adults: a pilot study. Gerontol Geriatr Med. 2016;2:2333721416678076.

30. Callahan CM, Unverzagt FW, Hui SL, Perkins AJ, Hendrie HC. Six-item screener to identify cognitive impairment among potential subjects for clinical research. Med Care. 2002:40(9):771-81.

31. Doble SE, Fisher AG. The dimensionality and validity of the older Americans resources and services (OARS) activities of daily living (ADL) scale. J Outcome Meas. 1998;2(1):4-24.

32. Bandura A. Health promotion by social cognitive means. Health Educ Behav. 2004;31(2):143-64.

33. Venkatesh V. Determinants of perceived ease ofUse: integrating control, intrinsic motivation, and emotion into the technology acceptance model. Inf Syst Res. 2000;11(4):342-68.

34. Siervo M, Bertoli S, Battezzati A, Wells JC, Lara J, Ferraris C, Tagliabue A. Accuracy of predictive equations for the measurement of resting energy expenditure in older subjects. Clin Nutr. 2014;33(4):613-9.

35. Riebe D, Franklin BA, Thompson PD, Garber CE, Whitfield GP, Magal M, Pescatello LS. Updating ACSM's recommendations for exercise Preparticipation health screening. Med Sci Sports Exerc. 2015;47(11):2473-9.

36. Garber CE, Blissmer B, Deschenes MR, Franklin BA, Lamonte MJ, Lee IM, Nieman DC, Swain DP. American College of Sports M: American College of Sports Medicine position stand. Quantity and quality of exercise for developing and maintaining cardiorespiratory, musculoskeletal, and neuromotor fitness in apparently healthy adults: guidance for prescribing exercise. Med Sci Sports Exerc. 2011;43(7):1334-59.
37. Garber CE, Blissmer B, Deschenes MR, Franklin BA, Lamonte MJ, Lee IM, Nieman DC, Swain DP. Quantity and Quality of Exercise for Developing and Maintaining Cardiorespiratory, Musculoskeletal, and Neuromotor Fitness in Apparently Healthy Adults: Guidance for Prescribing Exercise. Med Sci Sports Exerc. 2011;43(7):1334-59.

38. Borg GA. Psychophysical bases of perceived exertion. Med Sci Sports Exerc. 1982;14(5):377-81.

39. Piercy Katrina L, Troiano Richard P. Physical activity guidelines for Americans from the US Department of Health and Human Services. Circ Cardiovasc Qual Outcomes. 2018;11(11):e005263.

40. Nicklas BJ, Chmelo E, Delbono O, Carr JJ, Lyles MF, Marsh AP. Effects of resistance training with and without caloric restriction on physical function and mobility in overweight and obese older adults: a randomized controlled trial. Am J Clin Nutr. 2015;101(5):991-9.

41. Pahor M, Guralnik JM, Ambrosius WT, Blair S, Bonds DE, Church TS, Espeland MA, Fielding RA, Gill TM, Groessl EJ, et al. Effect of structured physical activity on prevention of major mobility disability in older adults: the LIFE study randomized clinical trial. JAMA. 2014;311(23):2387-96.

42. Stiggelbout M, Hopman-Rock M, Tak E, Lechner L, Van Mechelen W. Dropout from exercise programs for seniors: a prospective cohort study. J Aging Phys Act. 2005;13(4):409-21.

43. Hawley-Hague H, Horne M, Skelton DA, Todd C. Review of how we should define (and measure) adherence in studies examining older adults' participation in exercise classes. BMJ Open. 2016;6(6):e011560.

44. Prescott S, Traynor JP, Shilliday I, Zanotto T, Rush R, Mercer TH. Minimum accelerometer wear-time for reliable estimates of physical activity and sedentary behaviour of people receiving haemodialysis. BMC Nephrol. 2020;21(1):230.

45. Aadland E, Ylvisåker E. Reliability of the Actigraph GT3X+ accelerometer in adults under free-living conditions. PLoS One. 2015;10(8):e0134606.

46. Subar AF, Kirkpatrick SI, Mittl B, Zimmerman TP, Thompson FE, Bingley C, Willis G, Islam NG, Baranowski T, McNutt S, et al. The automated selfadministered 24-hour dietary recall (ASA24): a resource for researchers, clinicians and educators from the National Cancer Institute. J Acad Nutr Diet. 2012;112(8):1134-7.

47. Jensen MD, Ryan DH, Apovian CM, Ard JD, Comuzzie AG, Donato KA, Hu FB, Hubbard VS, Jakicic JM, Kushner RF, et al. 2013 AHA/ACC/TOS quideline for the Management of Overweight and Obesity in adults. Circulation. 2014 129(25 suppl 2):S102-38

48. Lauretani F, Russo CR, Bandinelli S, Bartali B, Cavazzini C, Di lorio A, Corsi AM, Rantanen T, Guralnik JM, Ferrucci L. Age-associated changes in skeletal muscles and their effect on mobility: an operational diagnosis of sarcopenia. J Appl Physiol. 2003;95(5):1851-60.

49. Jones CJ, Rikli RE, Beam WC. A 30-s chair-stand test as a measure of lower body strength in community-residing older adults. Res Q Exerc Sport. 1999; 70(2):113-9.

50. Zhang Q, Li YX, Li XL, Yin Y, Li RL, Qiao X, Li W, Ma HF, Ma WH, Han YF, et al. A comparative study of the five-repetition sit-to-stand test and the 30second sit-to-stand test to assess exercise tolerance in COPD patients. Int J Chron Obstruct Pulmon Dis. 2018;13:2833-9.

51. Petersen C, Steffen T, Paly E, Dvorak L, Nelson R. Reliability and minimal detectable change for sit-to-stand tests and the functional gait assessment for individuals with Parkinson disease. J Geriatr Phys Ther. 2017;40(4):223-6.

52. Wright AA, Cook CE, Baxter GD, Dockerty JD, Abbott JH. A comparison of 3 methodological approaches to defining major clinically important improvement of 4 performance measures in patients with hip osteoarthritis. J Orthop Sports Phys Ther. 2011;41(5):319-27.

53. Bohannon RW. Minimal clinically important difference for grip strength: a systematic review. J Phys Ther Sci. 2019;31(1):75-8.

54. Laboratories ATSCOPSFCPF. ATS statement: guidelines for the six-minute walk test. Am J Respir Crit Care Med. 2002;166(1):111-7.

55. Bohannon RW, Crouch R. Minimal clinically important difference for change in 6-minute walk test distance of adults with pathology: a systematic review. J Eval Clin Pract. 2017;23(2):377-81.

56. Sayers SP, Jette AM, Haley SM, Heeren TC, Guralnik JM, Fielding RA. Validation of the late-life function and disability instrument. J Am Geriatr Soc. 2004:52(9):1554-9.

57. Van Domelen DR. Accelerometry: functions for processing minute-to-minute accelerometer data. R Package version 3.1.2; 2018.

58. Vaportzis E, Clausen MG, Gow AJ. Older adults perceptions of technology and barriers to interacting with tablet computers: a focus group study. Front Psychol. 2017:8:1687 
59. Mody L, Miller DK, McGloin JM, Freeman M, Marcantonio ER, Magaziner J, Studenski S. Recruitment and retention of older adults in aging research. $J$ Am Geriatr Soc. 2008;56(12):2340-8.

60. Acton G. Health-promoting self-Care in Family Caregivers. West J Nurs Res. 2002;24:73-86

61. Bernard MA, Clayton JA, Lauer MS. Inclusion Across the Lifespan: NIH Policy for Clinical Research. JAMA 2018;320(15):1535-6.

62. Brown JD, Hales S, Evans TE, Turner T, Sword DO, O'Neil PM, Ballentine S, Lovelace O, DuBose-Morris RA. Description, utilisation and results from a telehealth primary care weight management intervention for adults with obesity in South Carolina. J Telemed Telecare. 2020;26(1-2):28-35. https:// doi.org/10.1177/1357633X18789562. Epub 2018.

63. Chung LM, Law QP, Fong SS, Chung JW, Yuen PP. A cost-effectiveness analysis of teledietetics in short-, intermediate-, and long-term weight reduction. J Telemed Telecare. 2015;21(5):268-75.

64. Huang JW, Lin YY, Wu NY. The effectiveness of telemedicine on body mass index: A systematic review and meta-analysis. J Telemed Telecare. 2019; 25(7):389-401. https://doi.org/10.1177/1357633X18775564. Epub 2018.

65. Rutledge T, Skoyen JA, Wiese JA, Ober KM, Woods GN. A comparison of MOVE! Versus TeleMOVE programs for weight loss in veterans with obesity. Obes Res Clin Pract. 2017;11(3):344-51.

66. Barnason S, Zimmerman L, Schulz P, Pullen C, Schuelke S. Weight management telehealth intervention for overweight and obese rural cardiac rehabilitation participants: A randomised trial. J Clin Nurs. 2019;28(9-10): 1808-18. https://doi.org/10.1111/jocn.14784. Epub 2019.

67. Hong J, Kim J, Kim SW, Kong HJ. Effects of home-based tele-exercise on sarcopenia among community-dwelling elderly adults: Body composition and functional fitness. Exp Gerontol. 2017;87(Pt A):33-9.

68. Haugen HA, Tran ZV, Wyatt HR, Barry MJ, Hill JO. Using telehealth to increase participation in weight maintenance programs. Obesity (Silver Spring). 2007;15(12):3067-77.

69. Labiris G, Panagiotopoulou EK, Kozobolis VP. A systematic review of teleophthalmological studies in Europe. Int J Ophthalmol. 2018;11(2):314-25.

70. de Swanepoel W, Hall JW 3rd. A systematic review of telehealth applications in audiology. Telemed J E Health. 2010;16(2):181-200.

71. Narasimha S, Madathil KC, Agnisarman S, Rogers H, Welch B, Ashok A, Nair A, McElligott J. Designing telemedicine Systems for Geriatric Patients: a review of the usability studies. Telemed e-Health. 2016;23(6):459-72.

72. Van Orden KA, Bower E, Lutz J, Silva C, Gallegos AM, Podgorski CA, Santos EJ, Conwell Y. Strategies to Promote Social Connections Among Older Adults During 'Social Distancing' Restrictions. Am J Geriatr Psychiatry. 2020. https://doi.org/10.1016/j.jagp.2020.1005.1004.

73. Fedele DA, Cushing CC, Fritz A, Amaro CM, Ortega A. Mobile health interventions for improving health outcomes in youth: a meta-analysis. JAMA Pediatr. 2017;171(5):461-9.

74. Romeo A, Edney S, Plotnikoff R, Curtis R, Ryan J, Sanders I, Crozier A, Maher C. Can smartphone apps increase physical activity? Systematic review and meta-analysis. J Med Internet Res. 2019;21(3):e12053.

75. Jakicic JM, Davis KK, Rogers RJ, King WC, Marcus MD, Helsel D, Rickman AD, Wahed AS, Belle SH. Effect of wearable technology combined with a lifestyle intervention on long-term weight loss: the IDEA randomized clinical trial. JAMA. 2016;316(11):1161-71

76. Spring B, Duncan JM, Janke EA, Kozak AT, McFadden HG, DeMott A, Pictor A, Epstein LH, Siddique J, Pellegrini CA, et al. Integrating technology into standard weight loss treatment: a randomized controlled trial. JAMA Intern Med. 2013;173(2):105-11.

77. McDermott MM, Spring B, Berger JS, Treat-Jacobson D, Conte MS, Creager MA, Criqui MH, Ferrucci L, Gornik HL, Guralnik JM, et al. Effect of a homebased exercise intervention of wearable technology and telephone coaching on walking performance in peripheral artery disease: the HONOR randomized clinical trial. JAMA. 2018;319(16):1665-76.

78. Hutchesson MJ, Rollo ME, Krukowski R, Ells L, Harvey J, Morgan PJ, Callister R, Plotnikoff R, Collins CE. eHealth interventions for the prevention and treatment of overweight and obesity in adults: a systematic review with meta-analysis. Obes Rev. 2015;16(5):376-92.

79. Patel ML, Wakayama LN, Bass MB, Breland JY. Motivational interviewing in eHealth and telehealth interventions for weight loss: a systematic review. Prev Med. 2019;126:105738

80. Internet Service in New Hampshire. 2020. https://www.pewresearch.org/ internet/chart/internet-use-by-age/. Accessed 1 Oct 2020.

\section{Publisher's Note}

Springer Nature remains neutral with regard to jurisdictional claims in published maps and institutional affiliations.
Ready to submit your research? Choose BMC and benefit from:

- fast, convenient online submission

- thorough peer review by experienced researchers in your field

- rapid publication on acceptance

- support for research data, including large and complex data types

- gold Open Access which fosters wider collaboration and increased citations

- maximum visibility for your research: over $100 \mathrm{M}$ website views per year

At BMC, research is always in progress.

Learn more biomedcentral.com/submissions 